\title{
Research on Identification Technology of Ship Radiated Noise Based on Spectral Entropy Characteristics
}

\author{
Lv Haitao, Yang Rijie, Kong Xiaopeng, Zheng Xiaoqing \\ Naval Aviation University,China \\ *Ivhaitaolht@sina.com
}

Keywords: signal identification, inter-frame spectral similarity, entropy characteristics

\begin{abstract}
There is a great difference between the inter-frame spectrum of ship radiated noise and marine biological noise, so we can identify the two kinds of signals with the difference of inter-frame spectral similarity. This paper describes the inter-frame spectral similarity of the two signals quantitatively with entropy characteristics. To reduce the instantaneous interferences, FFT transform should be done to the framing signals and the amplitude spectrum should be fitted, then calculating the spectral entropy. The amplitude of the spectrum should be taken the square into calculation to increase the differences of the consecutive frames. The result of calculation indicates that there is a great difference between the spectral entropy characteristics of ship radiated noise and marine biological noise, the entropy of ship radiated noise is steady, and the entropy of marine biological noise is unsteady. The difference of the two kinds of signals will be obvious if we take the variances of entropy as identifying characteristics.
\end{abstract}

\section{Introduction}

In the sea war, the detection of enemy ships and submarines mostly is based on the detection of their radiated acoustic signals. But the generation mechanism for ship radiated noise is complex, and the underwater acoustic channel is mutable, many effective identifying characteristics can only reflect the characteristic of a subclasses target. Because of the frequency of acoustic signals emitted by these vocal creatures is overlapped with the frequency of submarine radiated noise, when the sonar detects ships and submarines, the marine biological noise will also be detected, which will influence the performance impact of the detection of ships and submarines. In order to distinguish the ship radiated noise and marine biological noise, many scholars have done a lot of research. Li Yi et al. used the measured data to analyze the line spectrum characteristics of the speedboat in practical work, and put forward a new line spectrum extraction algorithm which is composed of the long sequence FFT, line spectrum extraction, wide band noise rejection, amplitude conversion and background equilibrium [1]. On the basis of studying the radiation noise extraction algorithm of ship, Jing Zhihong et al. proposed the screening method and the fuzzy reasoning classification method for the particularity of the radiation noise spectrum distribution of the ship, and carried out the classification experiment on the ship target [2]. Yang Xiangfeng et al. accurately extracted the continuous spectrum characteristics of the ship radiated noise signal by using the least squares curve fitting method. And the power spectrum characteristics of the ship radiated noise were obtained by combining the continuum characteristics and the line spectrum characteristics. Then, the BP neural network is used to classify and classify the two types of target noise [3]. Abel et al. used a statistical likelihood ratio to detect regions containing narrow band energy, and then used image expansion and corrosion algorithms to remove most of the random noise points and extract the trajectories of the ship radiated noise [4]. Xiao Bin et al. established a model of low and medium frequency ship structural noise, and realized source identification of ship noises from the standpoint of statistics [5]. The study of the above scholars has achieved some results, but because the radiated noise of different ship is very different, and the situation also has a great relationship, so the recognition effect has also been greatly affected.

This paper describes the inter-frame spectral similarity quantitatively with entropy characteristics, recognizing and distinguishing between ship radiated noise and marine biological noise. 


\section{Spectrum Analysis of the Two Kinds of Signals}

Ship radiated noise is mainly composed of mechanical noise, propeller noise and hydrodynamic noise. The generation mechanism and spectrum structure of the noise are different. Mechanical noise is the noise generated by the vibration of the mechanical equipment on board, which is the main component of the low frequency band of the ship's radiated noise, and it is closely related to the navigational status and the mechanical working condition of the ship. Propeller noise is a mixed noise, it has a common characteristic and source with mechanical noise and hydrodynamic noise. Hydrodynamic noise is produced by irregular, undulating water flowing through the ship's surface, and it is the result of hydrodynamic forces acting on ships. But in general, hydrodynamic noise is covered by mechanical noise and propeller noise. The sound of marine creature noise has a great relationship with its biological behavior. It will send different sound signals under different behaviors such as feeding, frightening, courtship and communicate. Therefore, the spectral structure of marine biological noise signal will change with the change of time, season and external environment factors.

The frequency dependence of ship radiated noise and marine biological noise is shown in Fig. 1 [6].

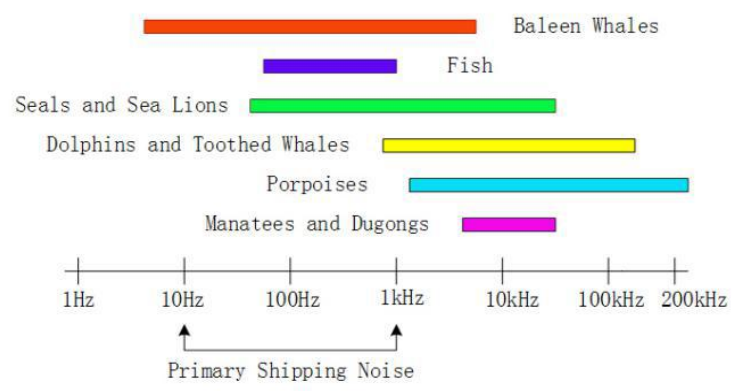

Figure 1. The frequency dependence diagram of ship radiated noise and marine biological noise

As can be seen from Fig. 1, the ship radiated noise and marine biological noise has a large overlap, and the two signals themselves are complex and volatile, so by means of conventional signal classification method is difficult to identify the two signals. Although the spectrum of different types of ships on different conditions vary widely, but in a short period of time, these factors which determine the ship noise spectrum will not change too much, so the spectrum of ship radiated noise in a short time will keep in a stable state, the adjacent two frames of the spectrum also have a certain similarity. While the marine biological noise tends to be short, the spectrum trend of the adjacent two frames changes greatly, and the inter-frame spectrum distribution is unstable. Fig. 2 shows the results of the spectral trend analysis of the measured two-frame noise signal for the measured ship radiated noise and marine ambient noise. It can be seen from Fig. 2 that the spectrum trend of the ship noise signal has good similarity, the target state is stable in a short time, and the inter-frame spectrum distribution of the marine biological noise signal has changed greatly. It has not the spectral trend of the similarity characteristics of the ship noise signal.

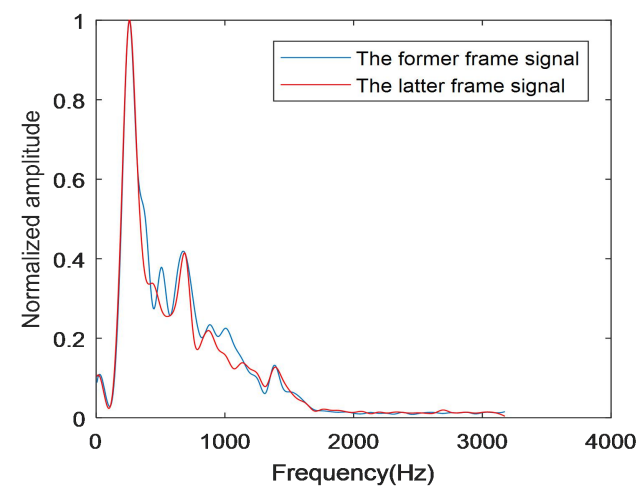

(a) Ship noise signal

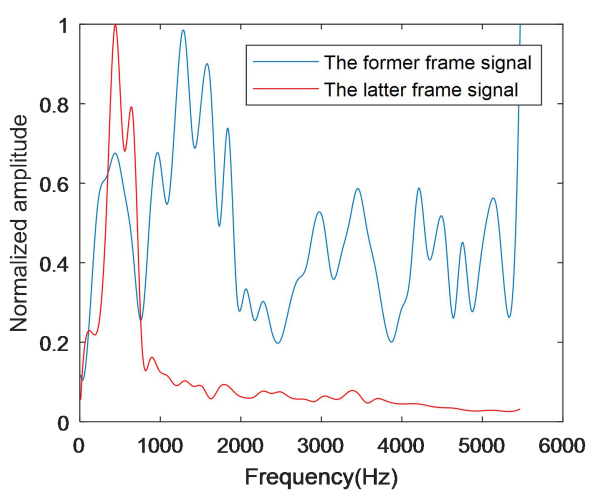

(b) Marine biological noise signal

Figure 2. Spectral trend graph of two adjacent noise signals 


\section{Inter-frame Spectral Similarity Feature Extraction}

There is a great difference between short-term inter-frame spectral similarity of ship radiated noise and marine biological noise, so short-term inter-frame spectral similarity can be taken as the identifying characteristics. The way to describe the differences of inter-frame spectral similarity quantitatively will directly affect the result of identification. The more accurate and reliable the way is, the more accurate and reliable the result of identification will be. This paper chooses entropy characteristic to describe the differences of inter-frame spectral similarity quantitatively.

Entropy can measure the amount of information in the signals. Suppose the probability density function(PDF) of every element in the continuous random variable $\boldsymbol{X}$ is $p_{X}\left(x_{i}\right)$, the entropy can be defined as:

$$
H(X)=-E_{x}\left[\log _{2} p_{X}\left(x_{i}\right)\right]=-\int_{-\infty}^{\infty} p_{X}\left(x_{i}\right) \cdot \log _{2} p_{X}\left(x_{i}\right) \cdot d x_{i}
$$

For the discrete random variable $\boldsymbol{x}$, its entropy can be defined as:

$$
H(\boldsymbol{x})=-E_{x}[\log p(\boldsymbol{x})]=-\sum_{i} p_{i}\left(x_{i}\right) \cdot \log p_{i}\left(x_{i}\right)
$$

Where, $p_{i}$ is the probability of element $x_{i}$, which belongs to the vector quantity $\boldsymbol{x}$, and $\sum_{i} p_{i}=1$, entropy is a kind of measurement of the uncertainty to a given distribution. In this paper, the trend of inter-frame spectral similarity of ship radiated noise is strong, it can be understood that if the spectral distribution trend of a frame is known, the next frame will obtain less new amount of information, the uncertainty of the spectral distribution trend from one frame to the next frame will be little. But the spectral distribution trend of marine biological noise is more difficult to estimate, because the former frame of marine biological noise signal can provide more amount of information, its entropy can be bigger relatively, so we can assess the similarity feature of the spectral trend of the adjacent two frames with entropy.

Suppose the target signal demodulated by airborne buoy information system is $\boldsymbol{x}$, the noise signal is divided into time slices including several time frames. Performing a fast Fourier transform(FFT) within the $\mathrm{N}$ points of every frame to get $\boldsymbol{F}(w)$ and fitting the amplitude spectrum of every frame to reduce the instantaneous interferences, then we can obtain the amplitude spectrum $\boldsymbol{f}(w)$ of every framing signal, so the spectral entropy $E n(x)$ of signal $\boldsymbol{x}$ can be calculated as:

$$
\begin{gathered}
p_{i, j}=f_{i, j}^{2}(w) / \sum_{j=0}^{m-1} f_{i, j}^{2}(w) \\
E n(i)=-\sum_{j=0}^{m-1}\left(p_{i, j} \cdot \log _{2} p_{i, j}\right) \\
V_{E n}=\frac{1}{n} \sum_{i=0}^{n} E n^{2}(i)-\left[\frac{1}{n} \sum_{i=0}^{n} E n(i)\right]^{2}
\end{gathered}
$$

Where, $i=1,2, \cdots, n, n=N / 2, j=1,2, \cdots, m, m$ is the quantity of frames in a time slice. Squaring the elements in every column vector of $\boldsymbol{f}(w)$ to increase the differences of the consecutive frames. Fig. 3 shows the spectral entropy of every slice calculated by the Eq. 4 . $\operatorname{En}(x)$ of marine biological noise is unsteady, so we utilize Eq. 5 to calculate its variance as the final identifying characteristics. Fig. 4 shows the spectral entropy's variance distribution of ship radiated noise and marine biological noise in a time slice, we can see that the difference of $V_{E n}$ between the two kinds of signals is obvious. 


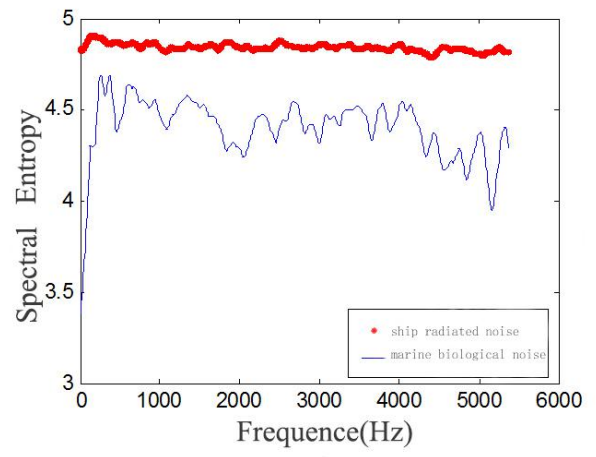

Figure 3. Spectral entropy of the two kinds of signals

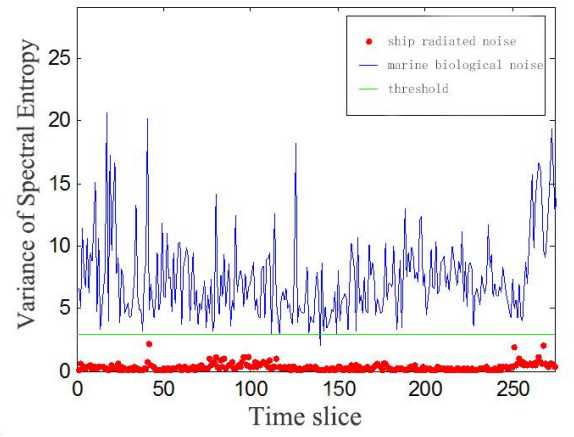

Figure 4. Variance of spectral entropy in short time slices

\section{The Recognition Results of the Two Kinds of Signals}

The similarity matrix of the two kinds of noise signals is calculated by using the similarity matrix calculation method [7]. The result is shown in Fig. 5. The gray scale value is used to represent the similarity of the spectral trend of the two frames in the signal. To make the difference display more obvious, the gray value is limited to 0 to 64 . The larger the gray value is, the greater the spectral similarity of the two frames is. From the spectral similarity matrix of the ship noise signal and the marine biological noise signal, it can be seen that the spectral grayscale value of the ship's noise signal is much higher than that of the marine biological noise signal, indicating that the short-term frame similarity of ship noise signal is better than that of marine biological noise signal. At the same time, from the histogram of the gray value of the two signals, the spectral similarity matrix of the ship noise signal is mainly distributed above 40, and the similarity matrix of the marine biological noise signal is in all ranges of 1 to 64 . This also shows that the use of similarity measurements can be a good distinction between ship noise signals and marine biological noise signals.

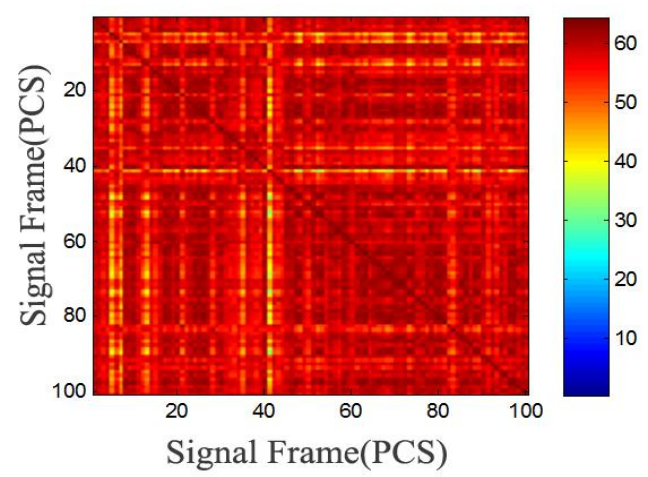

(a) The spectrum similarity matrix of ship noise signal

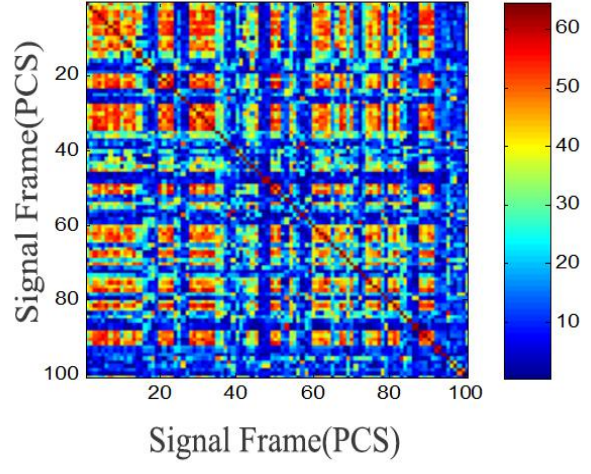

(b) The spectrum similarity matrix of marine biological noise signal

Figure 5. Spectral trend similarity matrix for noise signals

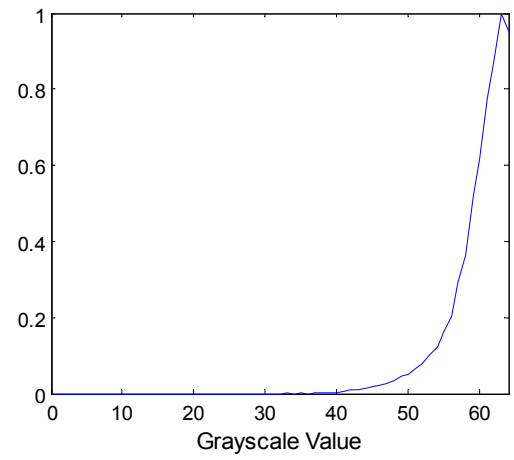

(a) The similarity matrix gray scale histogram of ship noise

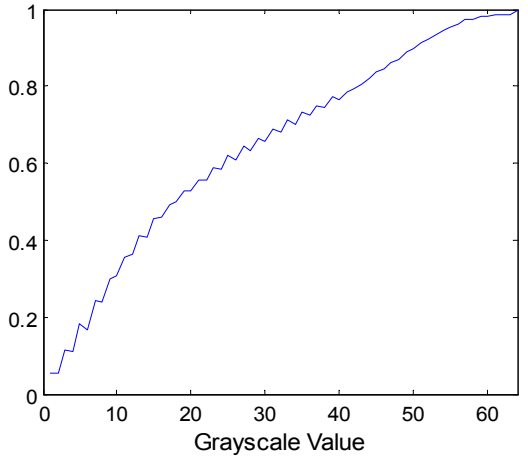

(b) The similarity matrix gray scale histogram of marine biological noise 


\section{Conclusion}

Based on the analysis of spectral characteristics of ship radiated noise signal and marine biological noise signal, a signal recognition method based on similarity of spectral distribution is proposed. The noise signal is divided into time slices including several time frames, and then the similarity of the inter-frame spectral distribution trends of the time frames in the fragments is calculated. Using the similarity matrix calculation method, the similarity matrix of the spectrum trend is calculated, and the difference of the characteristics of the ship radiated noise signal and the marine biological noise signal is more intuitively reflected. Then the variance of spectral entropy of the two kinds of signals is calculated, and the two kinds of signals are well classified and identified.

\section{References}

[1] Li Yi, Sun Changyu. Research on target noise and extraction of line-spectrums based on LOFAR[J]. Computer Engineering and Applications,2007,43:19-22.

[2] J.H. An, Y. Dodis and T. Rabin. 2002. On the security of joint signature and encryption, In Advances in Cryptology-Eurocrypt 2002, 2332: 83-107.Jing Zhihong, Xiang Dequan, Wang Yuanyi. A simulation experiment of underwater target identification based on linespectrum features[J]. Journal of System Simulation. 2000,12:642-644.

[3] Yang Xiangfeng, Zhang Xiaomin, Sun Jihong. A new power spectrum feature extractive method of ship radiated noise[J]. Torpedo Technology. 2006,14(1):35-38.

[4] Abel J S, Lee H J, and Lowell A P. An image processing approach to frequency tracking[R]. In ICASSP-92, II -561-564.

[5] Xiao Bin, Liu Wenshuai, Gao Chao, Liu Zhigang. Source identification methods of low and medium frequency ship structure noise[J]. Journal of Harbin Engineering University. 2015,36(12):1596-1602.

[6] Shi Guangzhi. Theoretical modeling and extracting fine structure of ship propeller cavitation noise modulation information[D]. Qingdao: Naval Submarine Academy, 2009.

[7] M.Cooper and J.Foote. Automatic music summarization via similarity analysis[C]. In Proc. $3^{\text {rd }}$ Int.Conf.Retriecal, Paris, France. 2002:81-85. 